\title{
Dusty early-type galaxies and passive spirals
}

\author{
Kate Rowlands, Loretta Dunne, Steve Maddox \& the \\ Herschel-ATLAS and GAMA collaborations
}

\author{
School of Physics \& Astronomy, The University of Nottingham, University Park Campus, \\ Nottingham, NG7 2RD, UK \\ email: ppxkr@nottingham.ac.uk
}

\begin{abstract}
Early-type galaxies (ETGs) are thought to be devoid of dust and star-formation, having formed most of their stars at early epochs. We present the detection of the dustiest ETGs in a large area blind submillimetre survey with Herschel (H-ATLAS, Eales et al. 2010), where the lack of pre-selection in other bands makes it the first unbiased survey for cold dust in ETGs. We compare to a control sample of optically selected ETGs to investigate how the two populations are different. We also highlight the properties of an interesting population of passive spirals detected by Herschel.
\end{abstract}

Keywords. galaxies: evolution, galaxies: elliptical and lenticular, cD, (ISM:) dust, extinction, infrared: galaxies, submillimeter

\section{Sample selection}

The parent sample of H-ATLAS galaxies in this study have a $5 \sigma$ detection at $250 \mu \mathrm{m}$, a reliable optical counterpart to the submillimetre source (see Smith et al. 2011a) and a spectroscopic redshift, many of which are taken from the GAMA survey (Driver et al. 2011). This gave rise to a total of 1087 galaxies, which were visually classified by eye using SDSS images into four categories: early-type, late-type, merger and unknown. ETGs were identified by looking for a dominant bulge and a complete lack of spiral arms, and latetypes were identified by the presence of spiral arms. Due to the shallow depth of the SDSS images, we do not discriminate between E and S0 types. In addition, a control sample of 1052 galaxies which were not detected at $250 \mu \mathrm{m}$ was constructed; these galaxies were chosen to have the same redshift and $r$-band magnitude distribution $(r, z)$ as the $\mathrm{H}$ ATLAS galaxies to eliminate selection effects between the samples. Further details can be found in Rowlands et al. (2011).

\section{SED fitting}

Physical parameters such as stellar mass, stellar population age and dust mass are derived for each galaxy using the multiwavelength SED fitting code of da Cunha, Charlot and Elbaz (2008). An energy balance argument is used to account for the UV-optical radiation from stars which is attenuated by dust and re-radiated in the far-infrared (FIR) and submillimetre. A Bayesian approach is used to derive statistical constraints on each physical parameter, as each observed galaxy SED is compared to a large library of models which encompass all plausible parameter combinations. For each galaxy, we build the marginalised likelihood distribution of any physical parameter by evaluating how well each model in the library can account for the observed properties of the galaxy (by computing the $\chi^{2}$ goodness of fit). This method ensures that possible degeneracies between model parameters are included in the final probability density function (PDF) of each parameter. An example SED with UV-submillimetre photometry is shown in Fig. 1. 


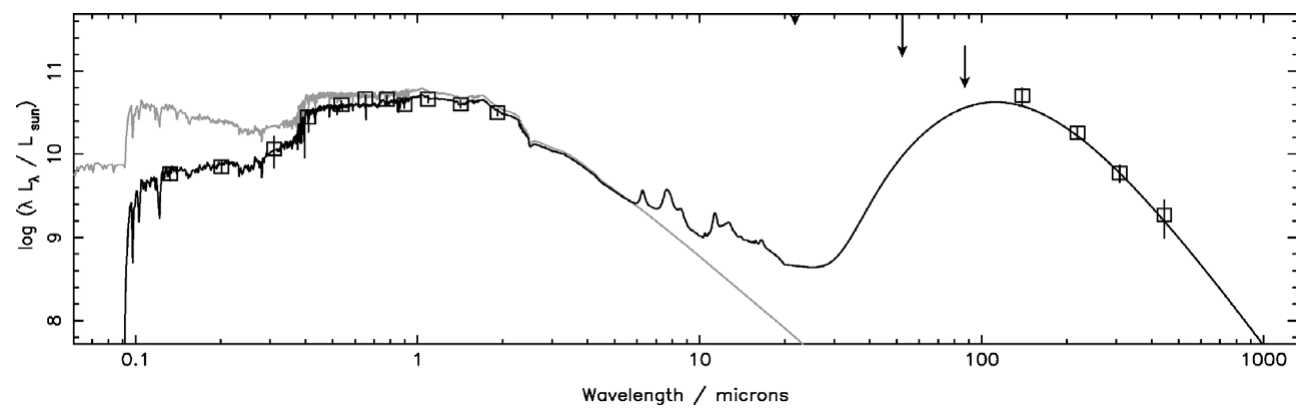

Figure 1. Example SED of an ETG, with observed photometry from the UV-submillimetre (squares), $5 \sigma$ upper limits are shown as arrows. Errors on the photometry are described in Smith et al. (2011b). The black line is the best fit model, and the grey line is the attenuated optical model.
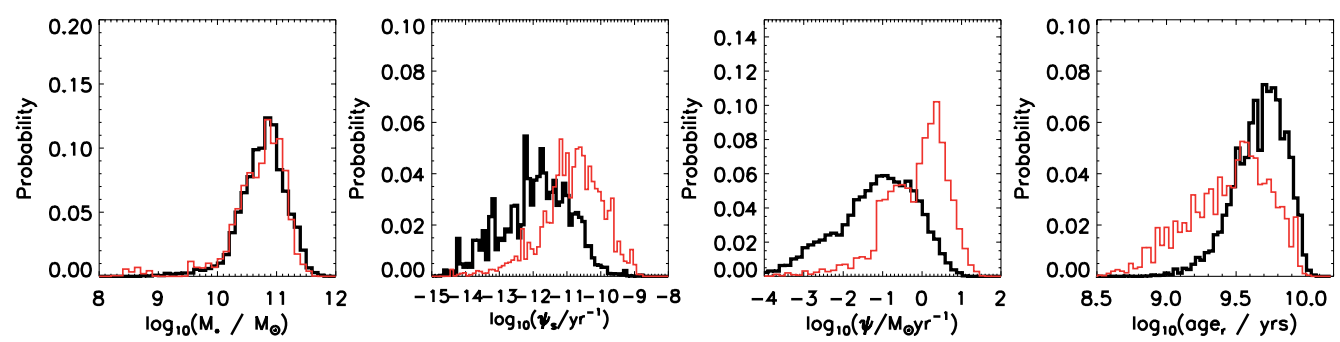

Figure 2. Average PDFs of the SED parameters of $42 \mathrm{H}$-ATLAS ETGs (thin line) compared to 222 control ETGs (thick line). The parameters are (from left to right): $\mathrm{M}_{*}$, stellar mass; $\psi_{\mathrm{S}} / \mathrm{yr}$, $\mathrm{SSFR} ; \psi / \mathrm{M}_{\odot} \mathrm{yr}^{-1}$, SFR; and age $\mathrm{r}$, the $r$-band light-weighted age of the stellar population.

\section{How are H-ATLAS ETGs different to control ETGs?}

A comparison of the average PDFs of the H-ATLAS and control ETGs in Fig. 2 shows that the stellar mass distributions are not significantly different, since the control sample is selected to have the same $r, z$ distribution. Conversely, on average the H-ATLAS ETGs are more than an order of magnitude dustier than the control ETGs. The dust mass of the 233 control ETGs inferred from stacking at optical positions on the $250 \mu \mathrm{m}$ map (see Bourne et al. 2011$)$ is $(0.8-4.0) \times 10^{6} \mathrm{M}_{\odot}$ for $25-15 \mathrm{~K}$ dust, whereas the mean dust mass of the $42 \mathrm{H}$-ATLAS ETGs derived from the SED fitting is $5.5 \times 10^{7} \mathrm{M}_{\odot}$. The range of $\mathrm{M}_{\mathrm{d}} / \mathrm{M}_{*}$ for the control ETGs is $(1.4-6.8) \times 10^{-5}$ for $25-15 \mathrm{~K}$ dust, and on average, the mean SSFR of the control ETGs is 1.1 dex lower than that of H-ATLAS ETGs. A similar trend is found when comparing the mean SFR of the ETGs. For our control ETGs the mean $r$-band light-weighted age of the stellar population is $1.8 \mathrm{Gyr}$ older than the H-ATLAS ETGs. The rest-frame $N U V-r$ colours of the control ETGs are 1.0 magnitudes redder than the H-ATLAS ETGs, and as they contain less dust their red colour must be due to an old stellar population. Control ETGs seem to inhabit higher density environments on average than the H-ATLAS ETGs, however the environments of the two populations are only different at the $1.8 \sigma$ level. We do not have strong evidence that environment has an affect on whether an ETG is dusty at low-intermediate densities, as a larger sample is needed to test this.

\section{Origin of dust in ETGs}

Dust in ETGs has two possible origins: internal sources from asymptotic giant branch (AGB) stars, or an external supply from mergers. Using the chemical evolution code 

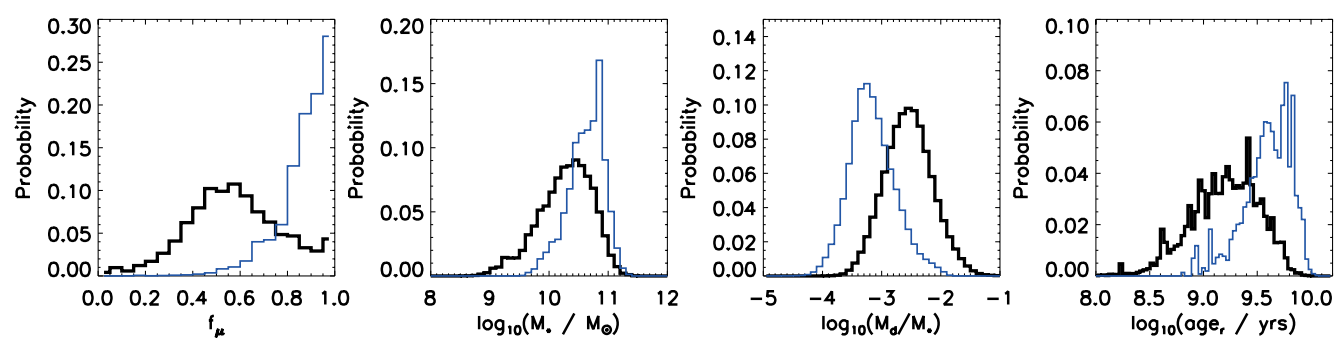

Figure 3. Average PDFs of the SED parameters of 19 passive spirals (SSFR $<10^{-11} / \mathrm{yr}$, thin line) compared to 431 normal spirals with SSFR $\geqslant 10^{-11} / \mathrm{yr}$ (thick line). The parameters are (from left to right): $f_{\mu}$, the fraction of total dust luminosity contributed by the diffuse ISM; $\mathrm{M}_{*}$, stellar mass; $\mathrm{M}_{\mathrm{d}} / \mathrm{M}_{*}$, dust to stellar mass ratio; and age $\mathrm{r}_{\mathrm{r}}$, the $r$-band light-weighted age of the stellar population.

of Gomez et al. (in prep), it is found that AGB stars cannot produce enough dust to account for that observed in the H-ATLAS ETGs. Even if dust production in Type II $\mathrm{SNe}$ is a significant dust source, the required dust injection rate for an average H-ATLAS ETG cannot be achieved. This indicates that an external source of dust from mergers is required, a substantial amount of dust grain growth must occur in the ISM or dust destruction is less efficient than predicted.

\section{Properties of passive spirals}

We also highlight the properties of a population of 19 passive spirals detected in $\mathrm{H}$ ATLAS. There is discussion in the literature whether some spirals are red due to dust extinction or an old stellar population (Wolf et al. 2009). By selecting a sample of HATLAS spirals with SSFR $<10^{-11} / \mathrm{yr}$ and comparing them to 'normal' spirals with SSFR $\geqslant 10^{-11} / \mathrm{yr}$, Fig. 3 shows that the majority of the dust luminosity is produced in the ISM $\left(f_{\mu}\right)$, which is heated by old stars. The passive spirals have higher stellar masses and less dust per stellar mass, meaning that the passive spirals are dust deficient compared to normal spirals. This could be a selection bias in that we can only detect dust in the most massive passive spirals as their $M_{d} / M_{*}$ ratio is much lower than the normal spiral population. Alternatively, it could be that passive galaxies tend to be massive (Masters et al. 2010). We find passive spirals have green-red rest-frame $N U V-r$ colours that are due to an old stellar population and not due to increased dust reddening.

\section{References}

Bourne, N. et al. MNRAS, submitted da Cunha, E., Charlot S., \& Elbaz, D. 2008, MNRAS, 388, 1595

Driver, S. et al. 2011, MNRAS, 413, 971

Eales, S. et al. 2010, PASP, 122, 499

Masters, K. et al. 2010, MNRAS, 405, 783

Rowlands, K. et al. 2011, MNRAS, in press

Smith, D. J. B. et al. 2011, MNRAS, 416, 857

Smith, D. J. B. et al. 2011, MNRAS, submitted

Wolf, C. et al. 2009, MNRAS, 393, 1302 